Submitted to Astronomical Journal

\title{
Optical Intra-day Variability in Blazars
}

\author{
Alok C. Gupta ${ }^{1}$, J. H. Fan ${ }^{2}$, J. M. Bai ${ }^{1}$ and S. J. Wagner ${ }^{3}$ \\ acgupta30@gmail.com
}

\begin{abstract}
We selected a sample of a dozen blazars which are the prime candidates for simultaneous multi-wavelength observing campaigns in their outburst phase. We searched for optical outbursts, intra-day variability and short term variability in these blazars. We carried out optical photometric monitoring of nine of these blazars in 13 observing nights during our observing run October 27, 2006 March 20, 2007 by using the 1.02 meter optical telescope equipped with CCD detector and BVRI Johnson broad band filters at Yunnan astronomical observatory, Kunming, China. From our observations, our data favor the hypothesis that three blazars: AO $0235+164$, S5 $0716+714$ and 3C 279 were in the outburst state; one blazar: 3C 454.3 was in the post outburst state; three blazars: S2 $0109+224$, PKS $0735+178$ and OJ 287 were in the pre/post outburst state; one blazar: ON 231 was in the low-state; and the state of one blazar: 1ES 2344+514 was not known because there is not much optical data available for the blazar to compare with our observations. We observed densely sampled 1534 image frames of these nine blazars. Out of three nights of observations of AO $0235+164$, intraday variability was detected in two nights. Out of five nights of observations of S5 0716+714, intra-day variability was detected in two nights. In one night of observations of PKS $0735+178$, intra-day variability was detected. Out of six nights of observations of $3 \mathrm{C} 454.3$, intra-day variability was detected in three nights. No intra-day variability was detected in S2 0109+224, OJ 287, ON 231, 3C 279 and 1ES $2344+514$ in their 1, 4, 1, 2 and 1 nights of observations respectively. AO 0235+164, S5 0716+714, OJ 287, 3C 279 and 3C 454.3 were observed
\end{abstract}

\footnotetext{
${ }^{1}$ National Astronomical Observatories/Yunnan Observatory, Chinese Academy of Sciences, P.O. Box 110, Kunming, Yunnan 650011, China.

${ }^{2}$ Center for Astrophysics, Guangzhou University, Guangzhou 510006, China

${ }^{3}$ Landessternwarte, Königstuhl 12, D - 69117, Heidelberg, Germany
} 
in more than one night and short term variations in all these blazars were also noticed. From our observations and the available data, we found that the predicted optical outburst with the time interval of $\sim 8$ years in AO $0235+164$ and $\sim 3$ years in S5 0716+714 have possibly occurred.

Subject headings: AGN: blazar: optical: observations - blazars

\section{Introduction}

Blazars represent a small subset of the most enigmatic class of radio-loud active galactic nuclei (AGN), exhibiting strong variability at all wavelengths of the whole electromagnetic (EM) spectrum, strong polarization from radio to optical wavelengths, and usually core dominated radio structures. The radiation of blazars at all wavelengths is predominantly nonthermal. This class includes BL Lacertae (BL Lac) objects and flat-spectrum radio quasars (FSRQs). BL Lacs show largely featureless optical continuum. In a unified model of the radio-loud AGN based on the angle between the line of sight and the emitted jet from the source, blazars jet make angle of $\sim 10^{\circ}$ from the line of sight (Urry \& Padovani 1995). The radiation emitted by the plasma, with bulk relativistic motion in the jet oriented at small viewing angles, is affected by relativistic beaming, which in turn implies a shortening of time scales by a factor $\delta^{-1}$, where $\delta$ is the Doppler factor.

From observations of blazars, it is known that they vary on the diverse time scales. Variability time scales of blazars can be broadly divided into 3 classes viz. intra-day variability (IDV) or micro-variability, short term outbursts and long term trends. Significant variations in flux of a few tenths of magnitude over the course of a day or less is often known as IDV (Wagner \& Witzel 1995). Short term outburst and long term trends can have time scales range from few weeks to several months and several months to years, respectively. In last about two decades, variability of blazars in radio to optical bands on diverse time scales have been reported in a large number of papers (e.g. Miller et al. 1989; Courvoisier, et al. 1995; Heidt \& Wagner 1996; Takalo et al. 1996; Sillanpää et al. 1996a, 1996b; Bai, et al. 1998, 1999; Fan et al. 1998, 2002, 2007; Xie, et al. 2002a; Gupta et al. 2004; Ciprini et al. 2003, 2007 and references therein).

We selected a sample of a dozen blazars which are prominent candidates for simultaneous multi-wavelength observing campaigns in their outburst phase. The motivation of the present work was to observe these blazars in search for IDV, short term variability and also find out if there is any one being in the outburst state. Blazar emission mechanism in the outburst state and detected IDV is strongly supported by the jet based models of radio-loud AGN. 
In general, blazar emission in the outburst state is nonthermal Doppler boosted emission from jets (Blandford \& Rees 1978; Marscher \& Gear 1985; Marscher et al. 1992, Hughes et al. 1992). There are other models of AGN that can explain the IDV in any type of AGN are optical flares, disturbances or hot spots on the accretion disk surrounding the black hole of the AGN (Mangalam \& Wiita 1993 and references therein). Models based on the instabilities on accretion disc are mainly supported blazars IDV when the blazar is in the low-state. When a blazar is in the low-state, any contribution from the jets if at all present, is very weak. Recently, it is noticed that, in the low luminosity AGN, accretion disk is radiatively inefficient (Chiaberge et al. 2006; Capetti et al. 2007). So, there will be an alternative way to explain the IDV in the low-state of blazars, in which a weak jet emission will be responsible for the IDV. With this motivation we recently carried out optical

photometric observations of the nine blazars: S2 0109+224, AO 0235+164, S5 0716+714, PKS 0735+178, OJ 287, ON 231, 3C 279 and 1ES 2344+514 in R passband and 3C 454.3 in $\mathrm{V}$ and $\mathrm{R}$ passbands.

The paper is arranged as follows: section 2 describes observations and data analysis method, in section 3 we mentioned our results, discussion and conclusion of the present work is reported in section 4 .

\section{Observations and Data Reductions}

The photometric observations of the blazars S2 0109+224, AO 0235+164, S5 0716+714, PKS 0735+178, OJ 287, ON 231, 3C 279 and 1ES 2344+514 in Johnson R passband and 3C 454.3 in Johnson $\mathrm{V}$ and $\mathrm{R}$ passbands were carried out using the CCD detector (1024 pixels $\times 1024$ pixels) mounted at f/13.3 Cassegrain focus of the 1.02 meter RC (Ritchey Chertien) optical telescope at Yunnan observatory, Kunming, China. These blazars were observed during October 27, 2006 to March 20, 2007 in 13 observing nights. Each pixel of the CCD detector projected on the sky corresponds to 0.38 arcsec in both the dimensions. The entire CCD chip covers $\sim 6.5 \times 6.5 \mathrm{arcmin}^{2}$ of the sky. The read out noise and gain of the CCD were 3.9 electrons and 4.0 electrons/ADU respectively. Throughout the observing run, the typical seeing was $\sim 1.8$ arcsec ranging from 1.5 to 2.0 arcsec. Several bias frames were taken intermittently in each observing night and twilight sky flats were taken in $\mathrm{V}$ and $\mathrm{R}$ passbands. Detail photometric observation log is given in the Table 1.

For each observing night we generated a master bias by taking median of all bias frames in the night. Master bias frame is subtracted from all the target image frames and flat field image frames of the night. Then master $\mathrm{V}$ and $\mathrm{R}$ passband flat field image frames were generated for the night by taking median of all flat field images in $\mathrm{V}$ and $\mathrm{R}$ passband 
respectively. Image processing or pre processing (bias subtraction, flat-fielding and cosmic rays removal) were done using standard routines in IRAF1 (Image Reduction and Analysis Facility) software. Photometric reduction or processing of the data were performed by aperture photometric technique using DAOPHOT II (Dominian Astronomical Observatory Photometry) software (Stetson 1987). Aperture photometry was carried out with concentric apertures of radii $8,11,15$ and 20 pixels. The data reduced with different aperture radii were found to be in good agreement. However, it was noticed that the best signal to noise ratio was obtained with the aperture radius of 15 pixels $(5.7 \operatorname{arcsec}$ slightly more than $3 \times$ of typical FWHM).

In our observations of all nine blazars, local standard stars were also always present in the observed image frames. We always observed three or more local standard stars in our every target blazar field. The magnitude of standard stars which we observed in the field of our observed blazars are given in Table 2. These standard stars were used to check the non variable characteristic of standard stars and then transformed the instrumental magnitude of the blazars to the standard magnitude. Finally we selected two standard stars from each blazar field and plotted their differential instrumental magnitude light curve by adding arbitrary offset for clarity in the plot with the light curves of blazars (calibrated magnitude). Photometric data of our blazars observations are provided in Table 42 .

\section{Results}

\subsection{Variability Detection Criterion}

Using aperture photometry of blazar and few standard stars in the blazar field, we determined the differential instrumental magnitude of blazar - standard star A, blazar standard star B and standard star A - standard star B. Finally we selected star A or B to do calibration of the blazar data. We determined observational scatter from blazar - standard star A $\sigma$ (BL - Star A), blazar - standard star B $\sigma$ (BL - Star B) and standard star A - standard star B $\sigma$ (Star A - Star B). The variability of target blazar is investigated by

\footnotetext{
${ }^{1}$ IRAF is distributed by the National Optical Astronomy Observatories, which are operated by the Association of Universities for Research in Astronomy, Inc., under cooperative agreement with the National Science Foundation.

${ }^{2}$ Here we provide photometric data for the blazars S2 0109+224, S5 0716+714, PKS 0735+178, ON 231 and 1ES 2344+514. Data of the blazars AO 0235+164, OJ 287, 3C 279 and 3C 454.3 will be stored in the WEBT (http://www.to.astro.it/blazars/webt) archive. For questions regarding their avalability, please contact the WEBT President Massimo Villata (villata@oata.inaf.it)
} 
using variability parameter C, introduced by Romero et al. (1999).

The variability parameter $\mathrm{C}$ is expressed as the average of $\mathrm{C}_{1}$ and $\mathrm{C}_{2}$

$$
C_{1}=\frac{\sigma(B L-S \operatorname{tar} A)}{\sigma(\operatorname{Star} A-S \operatorname{tar} B)} \quad \text { and } \quad \mathrm{C}_{2}=\frac{\sigma(\mathrm{BL}-\mathrm{StarB})}{\sigma(\mathrm{Star} \mathrm{A}-\mathrm{Star} \mathrm{B})}
$$

If $\mathrm{C}>2.57$, the confidence limit of variability is $99 \%$. The value of $\mathrm{C}$ by using the two standard stars for all the nine blazars for different observing nights are reported in Table 3.

Intra-day variability amplitude defined as (Heidt \& Wagner 1996)

$$
A=100 \times \sqrt{\left(A_{\max }-A_{\min }\right)^{2}-2 \sigma^{2}} \%
$$

where $\mathrm{A}_{\max }$ and $\mathrm{A}_{\min }$ are the maximum and minimum magnitude in the calibrated light curve of the blazar. $\sigma$ is the averaged measurement error of the blazar light curve.

\subsection{IDV and Short Term Variability of Individual Blazars}

\section{S2 $0109+224$}

S2 $0109+224$ is a BL Lac object which has featureless optical spectrum. The host galaxy of the source was unresolved in NTT observations (Falomo 1996) and also in K passband observations using UKIRT (Wright et al. 1998). Falomo (1996) suggested that the redshift of the blazar $\mathrm{z} \geq 0.4$ based on its optical appearance, and assuming $\mathrm{M}_{R}=-23.5$ for it, a value similar to that characterizing some galaxies located at few arcseconds north-east of the object. The blazar has shown optical flux variability on diverse time scales (Ciprini et al. 2003). The degree of optical polarization varied between 10\% to $30 \%$ (Takalo 1991; Valtaoja et al. 1993).

We observed S2 0109+224 on January 11, 2007 in R passband. The light curves of the blazar and differential instrumental magnitude (star D - star C1) with arbitrary offset are displayed in the bottom panel of Fig. 6. From the figure it is clear that the source has not shown IDV on one night of our observations. We also performed the IDV detection test described in the variability detection criterion section and found that the value of $\mathrm{C}$ is 1.07 , which also confirms the non detection of IDV in our observations. Ciprini et al. (2003) have done the multi band optical monitoring of the source for seven years $(1994-2002)$ and also

plotted its B passband historical light curve (1906 - 2002). 1994 - 2002 light curves of the source show the brightest and faintest states of the source in $\mathrm{R}$ passband $\sim 13.2$ and $\sim 16.5$ magnitudes. In the present observations, we observed the source $\approx 1.0$ magnitude fainter 
than its brightest state and $\approx 2.3$ magnitude brighter than its faintest state. Therefore, it appears likely that we have observed the source in its pre/post outburst state.

\section{$\mathrm{AO} 0235+164$}

AO $0235+164$ at (redshift $\mathrm{z}=0.94$ ) is classified as a BL Lac object by Spinrad \& Smith (1975). This blazar has been extensively observed in the recent past from radio to X-ray bands and has shown variations in all those bands on diverse time scales (Ghosh \& Soundararajaperumal 1995; Heidt \& Wagner 1996; Romero et al. 1997, 2000; Webb et al. 2000; Raiteri et al. 2001; Padovani et al. 2004; and references therein). It is one of the blazars which has shown very high polarization $\mathrm{P}_{V}=44 \%$ and $\mathrm{P}_{I R}=36.4 \%$ (Impey et al. 1982; Stickel et al. 1993; Fan \& Lin 1999). Using 25 years (1975 - 2000) of radio and optical data, Raiteri et al. (2001) predicated that the blazar should show a possible correlated periodic radio and optical outburst on the time scale of $5.7 \pm 0.5$ years that was expected in February - March 2004. An intense WEBT (Whole Earth Blazar Telescope) multi-wavelength observing campaign during 2003-2005 was organized and the predicted out burst could not be detected (Raiteri et al. 2005; 2006a; 2006b). Raiteri et al. (2006b) did analysis of the long term optical light curves and reported that the optical outburst may have a longer time scale $\sim 8$ years.

We observed AO 0235+164 on January 09 and 10, and March 19, 2007 in R passband. The light curve of the blazar and differential instrumental magnitude (star 1 - star 3 ) with an arbitrary offset are displayed in Fig. 1. From the figure it is seen that the source has shown IDV on January 10 and March 19, 2007. We also performed the IDV detection test and found that the value of $\mathrm{C}$ for January 09, January 10 and March 19 are 2.00, 4.14 and 3.73 respectively which confirms that the source has shown IDV in the observations of January 10 and March 19. The variability amplitude on January 10 and March 19 are $13.7 \%$ and 9.5\% respectively. Day to day variations are distinctly visible in Figs. 1 and 7. In Fig. 7 the filled circles show the day average magnitude of AO $0235+164$ and open circles show the day average of differential magnitude of two standard stars (star 1 and star 3) offset by the same arbitrary constant on all the three observing nights. Fig. 7 also shows the variability trend and if the source has reached even brighter state than January 10, it will be before March 19, 2007. From variability trend from observations show the source was still in outburst phase on March 19. Most interestingly, if we compare our Figs. 1 and 7 to the

long term light curve (1990 - 2005) of (Fig. 4 top panel of Raiteri et al. 2006b), we found that we have possibly detected the brightest state (outburst) of the source after around mid 1999 (previous optical outburst state). It is interesting that the interval between the last optical outburst in 1999 and the present detected possible outburst state is about 7.5 years, which is consistent with the prediction made by (Raiteri et al. 2006b). Our observations of 
the source was also the part of the late 2006 to March 2007 multi-wavelength campaign by WEBT. The multi-wavelength campaign results show the source was in the outburst state in this period (Raiteri et al. 2007b).

\section{S5 $0716+714$}

S5 $0716+714$ is one of the brightest BL Lac objects. The optical continuum is so featureless that all attempts made to determine its redshift have failed. The non detection of host galaxy first set a lower limit of $z>0.3$ (Wagner et al. 1996) and very recently z $>$ 0.52 (Sbarufatti et al. 2005). This object is among one of the most well studied BL Lac objects for variability studies in the whole EM spectrum on diverse time scales (e.g. Wagner et al. 1990; Heidt \& Wagner 1996; Ghisellini et al. 1997; Villata et al. 2000; Raiteri et al. 2003; Pian et al. 2005; Bach et al. 2005, 2006; Nesci et al. 2005; Ostorero et al. 2006; Montagni et al. 2006; Foschini et al. 2006; Wu et al. 2007; Fan et al. 2007 and references therein). Wagner \& Witzel (1995) reported that the duty cycle of the source is 1 which implies that the source is almost always in the active state. First optical polarization study of this source was done by Takalo et al. (1994). They found that the source shows high polarization $\sim 20 \%$ and intra-day polarization variability upto 3.5\%. Fan et al. (1997a) reported even much higher optical polarization up to $29 \%$ in the source.

Since 1994, people started observing this source intensely in optical bands. There are 4 major optical outbursts reported in the source, these are: at the beginning of 1995, in late 1997, in the fall of 2001 and in March 2004 (Raiteri et al. 2003; Foschini et al. 2006). These 4 outbursts give a possible period of long term variability of $\sim 3.0 \pm 0.3$ years.

We observed S5 0716+714 on January 10, 12; February 23; March 19 and 20; 2007 in $\mathrm{R}$ passband. The light curves of the blazar and differential instrumental magnitude (star 5 - star 3) with different arbitrary offset are displayed in different panels in Fig. 2. From Fig. 2, it is clear that the source has shown IDV on 2 nights (January 12 and February 23, 2007) out of 5 nights of observations. We also performed the IDV detection test and found that the value of C for January 10, January 12, February 23, March 19 and March 20, 2007 are $1.80,2.86,3.88,0.95$ and 1.56 respectively, which also confirms that the source has shown IDV on January 12 and February 23. The variability amplitude on January 12 and February 23, 2007 are $6.3 \%$ and $5.2 \%$ respectively. Day to day variations are also distinctly visible in the Figs. 2 and 7. In Fig. 7, filled circles show the day average magnitude of S5 0716+714 and open circles show the day average differential magnitude of star 5 and star 3 with the same arbitrary constant added for all 5 observing nights. Fig. 7 shows clearly the day to day variations in the source and also shows the variability trend in the source. The source was at $\mathrm{R}=12.58 \mathrm{mag}$ on January 12, 2007 which is comparable to the earlier outburst magnitude in $\mathrm{R}$ passband ( 12.75 mag in beginning of $1995 ; 12.6$ mag in late $1997 ; 12.55$ 
mag in the fall of 2001) (Raiteri et al. 2003). So, we have possibly detected the brightest state of the outburst phase in the source on January 12, 2007. If the source has reached even brighter state than January 12, it will be before February 23, 2007. If we compare the detected possible outburst in the present observations, it gives a period of the present outburst is after $\sim 2.8$ years from recent previous outburst in March 2004. It is consistent from the possible long term period of the source $3.0 \pm 0.3$ years.

\section{PKS 0735+178}

PKS $0735+178$ is an optically bright, highly variable source and classified as a BL Lac object (Carswell et al. 1974). The host galaxy of the source is unresolved in optical imaging. In the optical spectrum of PKS 0735+178, Carswell et al. (1974) did not find any emission line, but they noticed two sharp absorption features at $3981 \AA$ and $3991 \AA$ which they identified with the Mg II $\lambda 2798$ doublet at redshift $\mathrm{z}>0.424$ which sets a lower limit of its redshift. Very recently imaging redshift $z=0.424$ is reported for the source using HST snapshot image (Sbarufatti et al. 2005). The source has been extensively studied in radio and optical bands in search for variability. In search for long term variability, in about a century long optical data using Jurkevich method, periodic components of 13.8-14.2 years were found (Fan et al. 1997b; Qian \& Tao 2004). In a recent paper, Ciprini et al. (2007) has reported several short possible periods. There are several attempts to search for optical IDV and day to day variation in the source in last about one and half decades. Optical IDV upto 0.5 mag has been reported in the source on some occasions (e.g. Xie et al. 1992; Massaro et al. 1995; Fan et al. 1997b; Zhang et al. 2004). PKS 0735+178 has shown high degree of optical and near-infrared polarizations which varies from $1 \%$ to $30 \%$ (e.g Mead et al. 1990; Takalo 1991; Takalo et al. 1992; Valtaoja et al. 1991, 1993; Tommasi et al. 2001).

We observed PKS 0735+178 on January 11, 2007 in R passband. The light curves of the blazar and differential instrumental magnitude (star C4 - star C1) with arbitrary offset are displayed in the second panel from the bottom in Fig. 6. From the figure it is clear that the source has shown IDV on one night of our observations. We also performed the IDV detection test and found that the value of $\mathrm{C}$ is 4.00 , which also confirms that the source has shown IDV on one night of our observations. The observed variability amplitude is $4.9 \%$. Ciprini et al. (2007) have done the multi band optical monitoring of the source for more than ten years $(1993$ - 2004) and also plotted its B passband historical light curve (1906 - 2004). 1993 - 2004 light curve of the source shows the brightest and faintest states of the source in $\mathrm{R}$ passband $\sim 14.0$ and $\sim 17.0$ magnitudes. In the present observations, we

observed the source $\approx 1.7$ magnitude fainter than the brightest state and $\approx 1.3$ magnitude brighter than its faintest state. Therefore, it appears likely that we have observed the source in pre/post outburst state. 


\section{OJ 287}

Blazar OJ 287 at redshift $\mathrm{z}=0.306$ is one of the most extensively observed extragalactic object. In the long term optical light curve, it exhibits a $\sim 12$ year periodic outburst with a double-peaked maxima in its flux variations (Takalo et al. 1994; Pursimo et al. 2000). Sillanpää et al. (1988) explained the periodic outburst in the blazar with a binary black hole model. In radio to optical bands, the source has been studied for flux and polarization variability on diverse time scales by several groups (e.g. Jorstad et al. 2007; Hovatta et al. 2007; Zhang et al. 2007; Bach et al. 2007; Wu et al. 2006; Fan et al. 2006 and references therein). Takalo et al. (1994) have reviewed its observational properties from radio to X-ray bands.

We observed OJ 287 on October 29, November 17, November 18, 2006; and January 10, 2007 in $\mathrm{R}$ passband. The light curves of the blazar and differential instrumental magnitude (star 10 - star 11) with same arbitrary offset are displayed in Fig. 3. From the figure it is clear that the source has not shown IDV on any of the four nights of our observations. We also performed the IDV detection test and found that the value of $\mathrm{C}$ for October 29, November 17, November 18, 2006; and January 10, 2007 are 0.72, 1.00, 0.73 and 1.07 respectively, which also confirms that the source has not shown IDV on any of the 4 nights of our observations. In Fig. 7, filled circles show the day average magnitude of OJ 287 and open circles show the differential magnitude of star 10 and star 11 with the same arbitrary offset on all the 4 nights of observations. From Fig. 7, day to day variation in the source is clearly visible. Pursimo et al. (2000) have done the multi band optical monitoring of the source for about five years (1993 - 1998) and also plotted its V passband historical light curve for about a century long observations. $1993-1998$ light curve of the source shows the brightest and faintest states of the source in the $\mathrm{R}$ passband $\sim 13.5$ and $\sim 16.5$ magnitudes. In the present observations, we observed the source $\sim 14.6-15.0$ mag in $\mathrm{R}$ passaband which is $\approx 1.0$ magnitude fainter than the brightest state and $\approx 1.5$ magnitude brighter than its faintest state. Therefore, it appears likely that we have observed the source in pre/post outburst state. Our observations of the source were also the part of November 2006 multi-wavelength campaign by WEBT 3 .

\section{ON 231}

ON 231 at redshift $\mathrm{z}=0.102$ is classified as a BL Lac object (Browne 1971). Historical B passband light curve of the source (1935 - 1997) was plotted by Tosti et al. (1998). There are not many attempts to study the variability behavior of the source. Source has shown optical flux variations on diverse time scales ranging from few hours to several years (Smith \& Nair 1995; Xie et al. 1992).

\footnotetext{
${ }^{3}$ Detail information of the WEBT campaign is available at http://www.to.astro.it/blazars/webt/
} 
We observed ON 231 on January 11, 2007 in R passband. The light curves of the blazar and differential instrumental magnitude (star D - star A) with arbitrary offset are displayed in second panel from top in Fig. 6. From the figure it is clear that the source has not shown IDV on one night of our observations. We also performed the IDV detection test and found that the value of $\mathrm{C}$ is 1.06 , which also confirms that the source has not shown IDV on one night of our observations. Tosti et al. (1998) have done the multi band optical monitoring of the source for three years $(1994-1997)$ in its great outburst state. $1994-$ 1997 light curve of the source shows the brightest and faintest states in $\mathrm{R}$ passband $\approx 13.5$ and $\approx 15.0$ magnitudes. In the present observations, we observed the source at $\mathrm{R} \sim 15.0$ magnitude. Therefore, it appears likely that we have observed the source in the low-state which is comparable to the faintest state observed by Tosti et al. (1998).

\section{$3 \mathrm{C} 279$}

3C 279 is an optical violent variable quasar and has shown large optical variation of $\Delta \mathrm{B} \geq 6.7$ magnitude from archival plates of Harvard collection (Eachus \& Liller 1975). The most rapid optical variation $\Delta \mathrm{V}=1.17$ magnitude was reported in 40 min on May 22, 1996 (Xie et al. 1999). On another occasion the source brightened by $\sim 2.0$ magnitude within 24 hours (Webb et al. 1990). A large amplitude short term optical variability of $\Delta \mathrm{R}=0.91$ magnitude in 49 days (April to June 2001) has been reported earlier by (Xie et al. 2002b). It has shown very high optical polarization $\mathrm{P}=43.3 \pm 1.3 \%$ (Scarpa \& Falomo 1997). The source has also shown optical polarization variability $17 \%$ to $8 \%$ in three nights and intraday polarization is also detected (Andruchow et al. 2003). Using 27 years of near-infrared K band observations, Fan (1999) has reported that the source has shown 4.59 magnitude variations and a strong period of $7.1 \pm 0.44$ years.

We observed 3C 279 on January 12 and February 23, 2007 in R passband. The light curves of the blazar and differential instrumental magnitude (star 8 - star 1) with different arbitrary offset are displayed in Fig. 4. The top and bottom panels of Fig. 4 show the observations on January 12 and February 23, 2007 respectively. From the figure it is clear that the source has not shown IDV on both the nights (January 12, and February 23, 2007) of observations. We also performed the IDV detection test and found that the value of $\mathrm{C}$ for January 12 and February 23, 2007 are 1.0 and 1.0 respectively, which also confirms that the source has not shown IDV on both the nights of our observations. Day to day variations are distinctly visible in the Fig. 4. In Fig. 7, filled circles show the day average magnitude of 3C 279 and open circles show the differential magnitude of star 8 and star 1 with same arbitrary offset on both the nights of observations. Fig. 7 also shows the variability trend and if the source has reached even brighter state than January 12, it was before February 23, 2007. So, we have possibly observed the source in the outburst phase. We have detected 
large amplitude $\sim \Delta R=1.5$ magnitude variation in the source in the time span of 42 days which is much larger variation with previous $\Delta \mathrm{R}=0.91$ magnitude in 49 days (April to June 2001) reported by (Xie et al. 2002b). Our observations of the source was also the part of April 2006 - February 2007 multi-wavelength observing campaign by WEBT 4 in the outburst state of the source.

\section{C 454.3}

The blazar 3C 454.3 is a flat spectrum radio quasar at redshift $\mathrm{z}=0.859$. Long term optical and radio observations $(1966$ - 2005) were displayed by Villata et al. (2006). They detected an unprecedented optical outburst in (2004 - 2005) which lasted about a year in their multi wavelength observing campaign by WEBT. To further see the behavior of the source, two more multi-wavelength observing campaigns were organized by WEBT (Oct. 2005 - June 2007; July 2007 - ongoing). Our observations of the source was also the part of Oct. 2005 - June 2007 multi-wavelength observing campaign by WEBT.

We observed 3C 454.3 during Oct. 27 - 30, 2006 in V and R passbands and January 9-10, 2007 in R passband. The light curves of the blazar and differential instrumental magnitude (star 3 - star 4) with same arbitrary offset for all 6 nights observations in $\mathrm{R}$ passband are plotted in the panels in the bottom row of the figure 5. V passband light curves for all 4 nights observations with same arbitrary offset are plotted in the panels in the top row of the figure 5. We performed the IDV detection test and found that the value of $\mathrm{C}$ in $\mathrm{R}$ passband 2.46, 2.64, 2.42, 2.08, 3.83 and 1.77 for observations on October 27, October 28, October 29, October 30, 2006; January 09 and January 10, 2007 respectively. The value of $\mathrm{C}$ shows that source has shown IDV in R passband on 2 nights. The variability amplitude on October 28, 2006 and January 092007 were $5.1 \%$ and $17.1 \%$ respectively. The value of $\mathrm{C}$ in $\mathrm{V}$ passband 2.05, 1.79, 3.00 and 0.50 were obtained for observations on October 27, October 28, October 29 and October 30, 2006 respectively. The value of $\mathrm{C}$ shows that source has shown IDV in $\mathrm{V}$ passband on one night. The variability amplitude on October 29, 2006 was 11.6\%. The present observations of the source was in its post outburst phase (Raiteri et al. 2007a).

\section{ES $2344+514$}

1ES 2344+514 is classified as a BL Lac object based on lack of optical emission lines and CaII 'break strength' less than 25\% (Perlman et al. 1996). It was first detected in $\mathrm{TeV} \gamma$-rays $(>350 \mathrm{GeV}$ ) by the Whipple group (Catanese et al. 1998) and discovered among one of the first five $\mathrm{TeV}$ detected blazars. First attempt of its optical IDV and short term variability was made by Xie et al. (2002a) in 6 observing nights during January

\footnotetext{
${ }^{4}$ Detail information of the WEBT campaign is available at http://www.to.astro.it/blazars/webt/
} 
2000 to January 2001. They detect small amplitude IDV and short term variability in their observations.

We observed 1ES 2344+514 on January 12, 2007 in $\mathrm{R}$ passband. The light curves of the blazar and differential instrumental magnitude (star C2 - star C1) with arbitrary offset are displayed in the top panel in Fig. 6. From the figure it is clear that the source has not shown IDV on one night of our observations. We also performed the IDV detection test and found that the value of $\mathrm{C}$ is 1.44 , which also confirms the non detection of IDV in source in one night of our observations. There is not much optical data available for the source, so, it is difficult to know in which state was the source during our observations. This source will remain one of our prime target to search for optical variability on diverse time scales in near future observations.

\section{Discussion \& Conclusion}

From our observations, our data favor the hypothesis that three blazars: AO 0235+164, S5 0716+714 and 3C 279 were in the outburst state; one blazar: 3C 454.3 was in the post outburst state; three blazars: S2 0109+224, PKS 0735+178 and OJ 287 were in the pre/post outburst state; one blazar: ON 231 was in the low-state; and the state of one blazar: 1ES $2344+514$ was not known because there is not much optical data available for the blazar. The available statistics is not sufficient to draw strong conclusions.

Detected outburst phase in AO $0235+164$ possibly confirms the $\sim 8$ years period in optical bands which was predicted by Raiteri et al. (2006b). AO 0235+164 has shown IDV on two night out of 3 nights of observations. One night in which we did not detected any IDV, observations duration were very short and no IDV could be detected (if it has really occurred on that night). IDV can be broadly divided into intrinsic and extrinsic classes. Extrinsic IDV is caused by refractive interstellar scintillation and only relevant in the low-frequency radio observations. Another extrinsic cause of IDV is gravitational micro-lensing. Optical imaging and spectroscopic observations of the source have revealed foreground absorbing systems at $\mathrm{z}=0.524$ and $\mathrm{z}=0.851$ (Cohen et al. 1987; Nilsson et al. 1996). The flux of the source is contaminated and absorbed by foreground absorbing systems, the stars of which can act as gravitational micro-lenses. The reported optical IDV in AO $0235+164$ is well suited by the gravitational micro-lensing. Day to day variations are also noticed in our observations in the blazar.

Out of 5 nights observations of S5 0716+714, IDV is detected in 2 nights of observations. Day to day variations are distinctly visible in all 5 nights of observations which confirms the 
variability detection on short time scale. Detected outburst possibly confirm the long term periodic variation time scale of $3.0 \pm 0.3$ years. IDV reported in S5 $0716+714$ is intrinsic one. The dominantly fundamental model for intrinsic variability is shocks propagating through the jet (Blandford \& Königl 1979; Marscher \& Gear 1985). Models to explain intrinsic IDV are based on a relativistic shock propagating down a jet and interacting with irregularities in the flow (Marscher et al. 1992) or relativistic shocks changing directions in the jet (Nesci et al. 2005). Another related model involves non-axisymmetric bubbles carried outward in relativistic magnetized jets (Camenzind \& Krockenberger 1992). The IDV reported here in S5 $0716+714$ can be explained by any one of these models.

Out of 2 nights observations of 3C 279, IDV is not detected. Day to day variations are shown in our two nights of observations of the source which confirms the variability detection on short time scale. We detected short term variation of $\sim 1.5$ magnitude in $R$ passband in 42 days which is much larger than the earlier detected short term variation of 0.91 magnitude in 49 days (Xie et al. 2002b). When we observed 3C 279, the source was in outburst/high-state. In the densely sampled light curves in search for IDV in the source at outburst/high-state has not shown any IDV. No IDV detection in 3C 279 shows the jet emission do not have any irregularities in the jet flow, relativistic shocks directions have also not changed from the line of sight, no non-axisymmetric bubbles were carried outward in the relativistic magnetized jets.

Two other blazars: PKS 0735+178 and 3C 454.3 have shown IDV in our observations. 3C 454.3 was in the post outburst phase and PKS $0735+178$ was in the pre/post outburst phase during our observations. The IDV detected in these two blazars is well suited for shock-in-jet models described above. In 3C 454.3, we have also noticed the short term variability. No IDV was detected in S2 0109+224, OJ 287, ON 231 and 1ES 2344+514 in our observations. In OJ 287, we have noticed the short term variability.

We thankfully acknowledge the critical comments by the referee which helped us to improve the paper significantly. We are thankful to Prof. W. Yuan for careful reading of the manuscript. ACG and JMB gratefully acknowledge the financial support from the national Natural Science Foundation of China (grant nos. NSFC 10533050 and NSFC 10573030). JHF's work is supported by the national Natural Science Foundation of China (grant nos. NSFC 10573005 and NSFC 10633010). 


\section{REFERENCES}

Andruchow, I., Cellone, S. A., Romero, G. E., et al. 2003, A\&A, 409, 857

Bach, U., Krichbaum, T. P., Ros, E., et al. 2005, A\&A, 433, 815

Bach, U., Krichbaum, T. P., Kraus, A., et al. 2006, A\&A, 452, 83

Bach, U., Raiteri, C. M., Villata, M., et al. 2007, A\&A, 464, 175

Bai, J. M., Xie, G. Z., Li, K. H., et al. 1998, A\&AS, 132, 83

Bai, J. M., Xie, G. Z., Li, K. H., et al. 1999, A\&AS, 136, 455

Blandford, R. D., \& Königl, A. 1979, ApJ, 232, 34

Blandford, R. D., \& Rees, M. 1978, Physica Scripta, 17, 265

Browne, I. W. A. 1971, Nature, 231, 515

Capetti, A., Axon, D. J., Chiaberge, M., et al. 2007, A\&A, 471, 137

Carswell, R. F., Strittmatter, P. A., Williams, R. E. et al. 1974, ApJ, 190, L101

Camenzind, M. \& Krockenberger, M. 1992, A\&A, 255, 59

Catanese, M., Akerlof, C. W., Badran, H. M., et al. 1998, ApJ, 501, 616

Chiaberge, M., Gilli, R., Macchetto, F. D. et al. 2006, ApJ, 651, 728

Ciprini, S., Takalo, L. O., Tosti, G., et al. 2007, A\&A, 467, 465

Ciprini, S., Tosti, G., Raiteri, C. M., et al. 2003, A\&A, 400, 487

Cohen, R. D., Smith, H. E., Junkkarinen, V. T., \& Burbidge, E. M. 1987, ApJ, 318, 577

Courvoisier, T. J.-L, Blecha, A., Bouchet, P. et al. 1995, ApJ, 438, 108

Eachus, L. A. \& Liller, W. 1975, ApJ, 200, L61

Fan, J. H., Cheng, K. S., Zhang, L., \& Liu, C. H. 1997a, A\&A, 327, 947

Fan, J. H., Xie, G. Z., Lin, R. G., et al. 1997b, A\&AS, 125, 525

Fan, J. H., Xie, G. Z., Pecontal, E., et al. 1998, ApJ, 507, 173

Fan, J. H. 1999, MNRAS, 308, 1032 
Fan, J. H. \& Lin, R. G. 1999, ApJS, 121, 131

Fan, J. H., Lin, R. G., Xie, G. Z., et al. 2002, A\&A, 381, 1

Fan, J. H., Hua, T.-X., Yuan, Y.-H., et al. 2006, PASJ, 58, 945

Fan, J. H., Liu, Y., Yuan, Y. H., et al. 2007, A\&A, 462, 547

Falomo, R. 1996, MNRAS, 283, 241

Fiorucci, M., Tosti, G., \& Rizzi, N., 1998, PASP, 110, 105

Fiorucci, M. \& Tosti, G., 1996, A\&AS, 116, 403

Foschini, L., Tagliaferri, G., Pian, E., et al. 2006, A\&A, 455, 871

Ghisellini, G., Villata, M., Raiteri, C. M., et al. 1997, A\&A, 327, 61

Ghosh, K. K., \& Soundararajaperumal, S. 1995, ApJS, 100, 37

Gupta, A. C., Banerjee, D. P. K., Ashok, N. M., \& Joshi, U. C. 2004, A\&A, 422, 505

Heidt, J., \& Wagner, S. J., 1996, A\&A, 305, 42

Hovatta, T., Tornikoski, M., Lainela, M., et al. 2007, A\&A, 469, 899

Hughes, P. A., Aller, H. D. \& Aller, M. F. 1992, ApJ, 396, 469

Impey, C. D., Brand, P. W. J. L. \& Tapia, S. 1982, MNRAS, 198, 11

Jorstad, S. G., Marscher, A. P., Stevens, J. A., et al. 2007, AJ, 134, 799

Mangalam, A. V., \& Wiita, P. J., 1993, ApJ, 406, 420

Marscher, A. P., \& Gear, W. K. 1985, ApJ, 298, 114

Marscher, A. P., Gear, W. K. \& Travis, J. P., 1992, in Variability of Blazars, ed. E. Valtaoja, M. Valtonen, Cambridge University Press, p. 85

Massaro, E, Nesci, R., Parola, G. C. et al. 1995, A\&A, 299, 339

Mead, A. R. G., Ballard, K. R., Brand, P. W., et al. 1990, A\&AS, 83, 183

Miller, H. R., Carini, M. T., \& Goodrich, B. D. 1989, Nature, 337, 627

Montagni, F., Maselli, A., Massaro, E., et al. 2006, A\&A, 451, 435 
Nesci, R., Massaro, E., Rossi, C., et al. 2005, AJ, 130, 1466

Nilsson, K., Charles, P. A., Pursimo, T., et al. 1996, A\&A, 314, 754

Ostorero, L., Wagner, S. J., Gracia, J., et al. 2006, A\&A, 451, 797

Padovani, P., Costamante, L., Giommi, P., et al. 2004, MNRAS, 347, 1282

Perlman, E. S., Stocke, J. T., Schachter, J. F., et al. 1996, ApJS, 104, 251

Pian, E., Foschini, L., Beckmann, V., et al. 2005, A\&A, 429, 427

Pursimo, T., Takalo, L., Sillanpää, A., et al. 2000, A\&AS, 146, 141

Qian, B. \& Tao, J. 2004, PASP, 116, 161

Raiteri, C. M., Villata, M., Aller, H. D., et al. 2001, A\&A, 377, 396

Raiteri, C. M., Villata, M., Tosti, G., et al. 2003, A\&A, 402, 151

Raiteri, C. M., Villata, M., Ibrahimov, M. A., et al. 2005, A\&A, 438, 39

Raiteri, C. M., Villata, M., Kadler, M., et al. 2006a, A\&A, 452, 845

Raiteri, C. M., Villata, M., Kadler, M., et al. 2006b, A\&A, 459, 731

Raiteri, C. M., Villata, M., Larionov, V. M., et al. 2007a, A\&A, 473, 819

Raiteri, C. M., Villata, M., Larionov, V. M., et al. 2007b, A\&A, accepted

Romero, G. E., Combi, J. A., Benaglia, P., et al. 1997, A\&A, 326, 77

Romero, G. E., Cellone, S. A., \& Combi, J. A. 1999, A\&AS, 135, 477

Romero, G. E., Cellone, S. A., \& Combi, J. A. 2000, A\&A, 360, L47

Sbarufatti, B., Treves, A., \& Falomo, R., 2005, ApJ, 635, 173

Scarpa, R. \& Falomo, R. 1997, A\&A, 325, 109

Sillanpä̈̈, A., Haarala, S., Valtonen, M. J., et al. 1988, ApJ, 325, 628

Sillanpä̈̈, A., Takalo, L. O, Pursimo, T., et al. 1996a, A\&A, 305, 17L

Sillanpää, A., Takalo, L. O, Pursimo, T., et al. 1996b, A\&A, 315, 13L

Smith, P. S., Balonek, T. J., Heckert, P. A., et al. 1985, AJ, 90, 1184 
Smith, A. G. \& Nair, A. D. 1995, PASP, 107, 863

Spinrad, H., \& Smith, H. E., 1975, ApJ, 201, 275

Stetson, P. B. 1987, PASP, 99, 191

Stickel, M., Fried, J. M., \& Kühr, H. 1993, A\&AS, 98, 393

Takalo, L. O. 1991, A\&AS, 90, 161

Takalo, L. O., Sillanpä̈̈, A., Nilsson, K., et al. 1992, A\&AS, 94, 37

Takalo, L. O., Sillanpää, A., Nilsson, K., 1994, A\&AS, 107, 497

Takalo, L. O., Sillanpä̈̈, A., Pursimo, T., et al. 1996, A\&AS, 120, 313

Tommasi, L., Palazzi, E., Pian, E., et al. 2001, A\&A, 376, 51

Tosti, G., Fiorucci, M., Luciani, M., et al. 1998, A\&AS, 130, 109

Urry, C. M., \& Padovani, P. 1995, PASP, 107, 803

Valtaoja, L., Karttunen, H., Efimov, Y. S., Shakhovskoi, N. M. 1993, A\&A, 278, 371

Valtaoja, L., Sillanpä̈̈, A., Valtaoja, E. et al. 1991, AJ, 101, 78

Villata, M., Raiteri, C. M., Ghisellini, G. et al. 1997, A\&AS, 121, 119

Villata, M., Raiteri, C. M., Lanteri, L., et al. 1998, A\&AS, 130, 305

Villata, M., Mattox, J. R., Massaro, E., et al. 2000, A\&A, 363, 108

Villata, M., Raiteri, C. M., Balonek, T. J., et al. 2006, A\&A, 453, 817

Wagner, S. J., Sanchez-Pons, F., Quirrenbach, A., \& Witzel, A. 1990, A\&A, 235, L1

Wagner, S. J., \& Witzel, A. 1995, ARA\&A, 33, 163

Wagner, S. J., Witzel, A., \& Heidt, J. et al. 1996, AJ, 111, 2187

Webb, J. R., Carini, M. T., Clements, S., et al. 1990, AJ, 100, 1452

Webb, J. R., Howard, E., Benitez, E., et al. 2000, AJ, 120, 41

Wright, S. C., McHardy, I. M., Abraham, R. G., et al. 1998, MNRAS, 296, 961

Wu, J., Zhou, X., Wu, X.-B., et al. 2006, AJ, 132, 1256 
Wu, J., Zhou, X., Ma, J., et al. 2007, AJ, 133, 1599

Xie, G. Z., Li, H. K., Liu, F. K., et al. 1992, ApJS, 80, 683

Xie, G. Z., Li, H. K., Zhang, X. et al. 1999, ApJ, 522, 846

Xie, G. Z., Zhou, S. B., Dai, B. Z., et al. 2002a, MNRAS, 329, 689

Xie, G. Z., Liang, E. W., Zhou, S. B., et al. 2002b, MNRAS, 334, 459

Zhang, X., Zhang, L., Zhou, G., et al. 2004, AJ, 128, 1929

Zhang, X., Zhao, G., Zheng, Y. G., et al. 2007, AJ, 133, 1995 
Table 1. Complete log of optical photometric observations of nine blazars from 1.02 meter Yunnan Astronomical Observatory Telescope, Kunming, China.

\begin{tabular}{|c|c|c|c|c|c|c|c|}
\hline Blazar Name & $\alpha_{2000.0}$ & $\delta_{2000.0}$ & $\mathrm{z}$ & $\begin{array}{c}\text { Date of } \\
\text { Observations } \\
\text { dd.mm.yyyy }\end{array}$ & Filters & $\begin{array}{l}\text { Data } \\
\text { Points }\end{array}$ & $\begin{array}{c}\text { Duration } \\
\text { (hour) }\end{array}$ \\
\hline S2 $0109+224$ & 011205.7 & +224439.2 & $\geq 0.4$ & 11.01 .2007 & $\mathrm{R}$ & 80 & 3.00 \\
\hline \multirow[t]{3}{*}{ AO $0235+164$} & 023838.9 & +163659.3 & $\overline{0} .94$ & 09.01 .2007 & $\mathrm{R}$ & 4 & 0.26 \\
\hline & & & & 10.01 .2007 & $\mathrm{R}$ & 79 & 3.04 \\
\hline & & & & 19.03 .2007 & $\mathrm{R}$ & 6 & 0.40 \\
\hline \multirow{5}{*}{ S5 $0716+714$} & 072153.5 & +712036.4 & $0.3 ?, 0.52 ?$ & 10.01 .2007 & $\mathrm{R}$ & 116 & 3.03 \\
\hline & & & & 12.01 .2007 & $\mathrm{R}$ & 185 & 3.56 \\
\hline & & & & 23.02 .2007 & $\mathrm{R}$ & 60 & 1.00 \\
\hline & & & & 19.03 .2007 & $\mathrm{R}$ & 82 & 1.58 \\
\hline & & & & 20.03 .2007 & $\mathrm{R}$ & 195 & 4.24 \\
\hline PKS $0735+178$ & 073807.4 & +174219.0 & 0.424 & 11.01 .2007 & $\mathrm{R}$ & 90 & 3.88 \\
\hline \multirow[t]{4}{*}{ OJ 287} & 085448.9 & +200631.0 & 0.306 & 29.10 .2006 & $\mathrm{R}$ & 15 & 1.14 \\
\hline & & & & 17.11 .2006 & $\mathrm{R}$ & 18 & 1.18 \\
\hline & & & & 18.11 .2006 & $\mathrm{R}$ & 16 & 1.06 \\
\hline & & & & 10.01 .2007 & $\mathrm{R}$ & 49 & 1.79 \\
\hline ON 231 & $12: 21: 31.7$ & $+28: 13: 58.5$ & 0.102 & 11.01 .2007 & $\mathrm{R}$ & 72 & 3.24 \\
\hline \multirow[t]{2}{*}{ 3C 279} & 125611.2 & -054721.5 & 0.5362 & 12.01 .2007 & $\mathrm{R}$ & 208 & 4.00 \\
\hline & & & & 23.02 .2007 & $\mathrm{R}$ & 100 & 2.35 \\
\hline \multirow[t]{6}{*}{ 3C 454.3} & 225357.7 & +160854.0 & 0.859 & 27.10 .2006 & $\mathrm{R}, \mathrm{V}$ & 15,9 & 1.59 \\
\hline & & & & 28.10 .2006 & $\mathrm{R}, \mathrm{V}$ & 15,8 & 1.27 \\
\hline & & & & 29.10 .2006 & $\mathrm{R}, \mathrm{V}$ & 15,10 & 1.76 \\
\hline & & & & 30.10 .2006 & $\mathrm{R}, \mathrm{V}$ & 14,2 & 1.10 \\
\hline & & & & 09.01.2007 & $\mathrm{R}$ & 11 & 0.68 \\
\hline & & & & 10.01 .2007 & $\mathrm{R}$ & 7 & 0.54 \\
\hline $1 \mathrm{ES} 2344+514$ & 234704.8 & +514217.9 & 0.044 & 12.01 .2007 & $\mathrm{R}$ & 53 & 1.89 \\
\hline
\end{tabular}


Table 2. Standard stars in the observed blazars field.

\begin{tabular}{|c|c|c|c|}
\hline Blazar Name & $\begin{array}{l}\text { Star } \\
\text { No. }\end{array}$ & $\begin{array}{l}\mathrm{R} \text { magnitude } \\
\quad(\text { error })\end{array}$ & Reference $^{a}$ \\
\hline \multirow[t]{4}{*}{ S2 0109+224 } & $\mathrm{I}$ & $12.11(0.04)$ & 1 \\
\hline & $\mathrm{C} 1$ & $14.72(0.06)$ & 1 \\
\hline & $\mathrm{D}$ & $14.09(0.05)$ & 1 \\
\hline & $\mathrm{E}$ & $14.94(0.05)$ & 1 \\
\hline \multirow[t]{5}{*}{$\mathrm{AO} 0235+164$} & 1 & $12.69(0.02)$ & 2 \\
\hline & 2 & $12.23(0.02)$ & 2 \\
\hline & 3 & $12.48(0.03)$ & 2 \\
\hline & 6 & $13.64(0.04)$ & 3 \\
\hline & $\mathrm{C} 1$ & $14.23(0.05)$ & 3 \\
\hline \multirow[t]{5}{*}{ S5 $0716+714$} & 2 & $11.12(0.01)$ & 4 \\
\hline & 3 & $12.06(0.01)$ & 4 \\
\hline & 5 & $13.18(0.01)$ & 4 \\
\hline & 6 & $13.26(0.01)$ & 4 \\
\hline & 8 & $13.79(0.02)$ & 4 \\
\hline \multirow[t]{5}{*}{ PKS $0735+178$} & $\mathrm{C}$ & $13.85(0.04)$ & 5 \\
\hline & $\mathrm{C} 1$ & $12.89(0.04)$ & 5 \\
\hline & $\mathrm{C} 2$ & $12.79(0.04)$ & 5 \\
\hline & $\mathrm{C} 4$ & $13.80(0.04)$ & 5 \\
\hline & $\mathrm{C} 7$ & $14.70(0.06)$ & 5 \\
\hline \multirow[t]{5}{*}{ OJ 287} & $\mathrm{C} 1$ & $15.50(0.07)$ & 6 \\
\hline & $\mathrm{C} 2$ & $15.66(0.08)$ & 6 \\
\hline & 4 & $13.74(0.04)$ & 6 \\
\hline & 10 & $14.34(0.05)$ & 6 \\
\hline & 11 & $14.65(0.05)$ & 6 \\
\hline \multirow[t]{3}{*}{ ON 231} & $\mathrm{~A}$ & $11.72(0.04)$ & 6 \\
\hline & $\mathrm{C} 1$ & $16.03(0.10)$ & 6 \\
\hline & $\mathrm{D}$ & $13.86(0.04)$ & 6 \\
\hline \multirow[t]{3}{*}{$3 \mathrm{C} 279$} & 1 & $12.05(0.02)$ & 7 \\
\hline & 5 & $15.47(0.02)$ & 7 \\
\hline & 8 & $14.44(0.00)$ & 8 \\
\hline \multirow[t]{8}{*}{ 3C 454.3} & 1 & $13.15(0.02)$ & 7 \\
\hline & 2 & $13.19(0.02)$ & 7 \\
\hline & 3 & $14.00(0.02)$ & 7 \\
\hline & 4 & $14.79(0.02)$ & 7 \\
\hline & 5 & $14.83(0.02)$ & 7 \\
\hline & 6 & $14.83(0.03)$ & 7 \\
\hline & 7 & $14.94(0.02)$ & 7 \\
\hline & 8 & $15.34(0.02)$ & 7 \\
\hline \multirow[t]{3}{*}{ 1ES $2344+514$} & $\mathrm{C} 1$ & $12.25(0.04)$ & 3 \\
\hline & $\mathrm{C} 2$ & $14.20(0.05)$ & 3 \\
\hline & $\mathrm{C} 2$ & $15.40(0.08)$ & 3 \\
\hline Blazar & $\begin{array}{l}\text { Star } \\
\text { No. }\end{array}$ & $\begin{array}{l}\mathrm{V} \text { magnitude } \\
(\text { error })\end{array}$ & Reference $^{a}$ \\
\hline 3C 454.3 & 1 & $13.71(0.02)$ & 7 \\
\hline
\end{tabular}


Table 2-Continued

\begin{tabular}{cccc}
\hline \hline Blazar Name & $\begin{array}{c}\text { Star } \\
\text { No. }\end{array}$ & $\begin{array}{c}\text { R magnitude } \\
\text { (error) }\end{array}$ & Reference $^{a}$ \\
\hline 2 & $13.80(0.02)$ & 7 \\
3 & $14.44(0.02)$ & 7 \\
4 & $15.21(0.02)$ & 7 \\
5 & $15.30(0.02)$ & 7 \\
6 & $15.34(0.03)$ & 7 \\
7 & $15.74(0.02)$ & 7 \\
8 & $15.94(0.02)$ & 7 \\
\hline
\end{tabular}

${ }^{a} 1$. Ciprini et al. 2003; 2. Smith et al. 1985; 3. Fiorucci et al. 1998; 4. Villata et al. 1998; 5. Ciprini et al. 2007; 6. Fiorucci et al. 1996; 7. Raiteri et al. 1998; 9. Villata et al. 1997 
Table 3. Results of IDV observations of the blazars. V and NV in the Variable column represent variable and non variable respectively. $\mathrm{N}$ represent the number of data points.

\begin{tabular}{|c|c|c|c|c|c|c|c|c|c|c|}
\hline $\begin{array}{c}\text { Date } \\
\text { dd.mm.yyyy }\end{array}$ & $\begin{array}{l}\text { Blazar } \\
\text { Name }\end{array}$ & $\begin{array}{c}\text { Time Range } \\
\text { UT }\end{array}$ & Filter & $\mathrm{N}$ & $\sigma_{\left(B L-S_{A}\right)}$ & $\sigma_{\left(B L-S_{B}\right)}$ & $\sigma_{\left(S_{A}-S_{B}\right)}$ & Variable & $\mathrm{C}$ & $\mathrm{A} \%$ \\
\hline 11.01 .2007 & S2 $0109+224$ & $11.36-14.35$ & $\mathrm{R}$ & 80 & 0.007 & 0.008 & 0.007 & NV & 1.07 & - \\
\hline 10.01 .2007 & & $13.10-16.14$ & $\mathrm{R}$ & 79 & 0.029 & 0.029 & 0.007 & $\mathrm{~V}$ & 4.14 & 13.7 \\
\hline 19.03.2007 & & $11.96-12.36$ & $\mathrm{R}$ & 6 & 0.040 & 0.042 & 0.011 & $\mathrm{~V}$ & 3.73 & 9.5 \\
\hline 10.01 .2007 & S5 $0716+714$ & $16.34-19.37$ & $\mathrm{R}$ & 116 & 0.009 & 0.009 & 0.005 & NV & 1.80 & - \\
\hline 19.03.2007 & & $12.60-14.18$ & $\mathrm{R}$ & 82 & 0.006 & 0.013 & 0.010 & NV & 0.95 & - \\
\hline 20.03.2007 & & $11.99-16.25$ & $\mathrm{R}$ & 195 & 0.023 & 0.027 & 0.016 & NV & 1.56 & - \\
\hline 11.01 .2007 & PKS $0735+178$ & $14.57-18.45$ & $\mathrm{R}$ & 90 & 0.012 & 0.012 & 0.003 & $\mathrm{~V}$ & 4.00 & 4.9 \\
\hline 29.10 .2006 & OJ 287 & $19.58-20.72$ & $\mathrm{R}$ & 15 & 0.027 & 0.044 & 0.049 & NV & 0.72 & - \\
\hline 17.11 .2006 & & $21.58-22.76$ & $\mathrm{R}$ & 18 & 0.006 & 0.008 & 0.007 & NV & 1.00 & - \\
\hline 18.11 .2006 & & $21.69-22.75$ & $\mathrm{R}$ & 16 & 0.013 & 0.028 & 0.028 & NV & 0.73 & - \\
\hline \multirow[t]{2}{*}{27.10 .2006} & 3C 454.3 & $11.76-13.10$ & $\mathrm{R}$ & 15 & 0.036 & 0.028 & 0.013 & NV & 2.46 & - \\
\hline & & $12.05-13.35$ & $\mathrm{~V}$ & 9 & 0.022 & 0.023 & 0.011 & NV & 2.05 & - \\
\hline \multirow[t]{2}{*}{28.10 .2006} & & $15.06-16.33$ & $\mathrm{R}$ & 15 & 0.018 & 0.019 & 0.007 & $\mathrm{~V}$ & 2.64 & 5.1 \\
\hline & & $15.44-16.11$ & $\mathrm{~V}$ & 8 & 0.041 & 0.034 & 0.021 & NV & 1.79 & - \\
\hline \multirow[t]{2}{*}{29.10 .2006} & & $11.15-12.54$ & $\mathrm{R}$ & 15 & 0.014 & 0.015 & 0.006 & NV & 2.42 & - \\
\hline & & $11.44-12.90$ & $\mathrm{~V}$ & 10 & 0.042 & 0.036 & 0.013 & $\mathrm{~V}$ & 3.00 & 11.6 \\
\hline \multirow[t]{2}{*}{30.10 .2006} & & $11.56-12.66$ & $\mathrm{R}$ & 14 & 0.013 & 0.012 & 0.006 & NV & 2.08 & - \\
\hline & & $11.86-11.95$ & $\mathrm{~V}$ & 2 & 0.013 & 0.000 & 0.013 & NV & 0.50 & - \\
\hline 09.01 .2007 & & $11.62-12.30$ & $\mathrm{R}$ & 11 & 0.059 & 0.056 & 0.015 & $\mathrm{~V}$ & 3.83 & 17.1 \\
\hline 10.01 .2007 & & $11.95-12.49$ & $\mathrm{R}$ & 7 & 0.054 & 0.077 & 0.037 & NV & 1.77 & - \\
\hline 12.01 .2007 & 1ES $2344+514$ & $11.61-13.50$ & $\mathrm{R}$ & 53 & 0.010 & 0.013 & 0.008 & NV & 1.44 & - \\
\hline
\end{tabular}


Table 4. R passband Photometric data of observed blazars. Complete table will be publish electronically.

\begin{tabular}{cc}
\hline \hline & \\
\hline Blazar Name & Date \\
S2 0109+224 & 11.01 .2007 \\
\hline UT & Magnitude \\
\hline 11.35889 & 14.148 \\
11.39917 & 14.155 \\
11.43583 & 14.142 \\
11.47250 & 14.145 \\
11.51083 & 14.139 \\
11.54833 & 14.144 \\
11.58556 & 14.138 \\
11.62194 & 14.140 \\
11.65917 & 14.140 \\
11.69639 & 14.144 \\
\hline
\end{tabular}




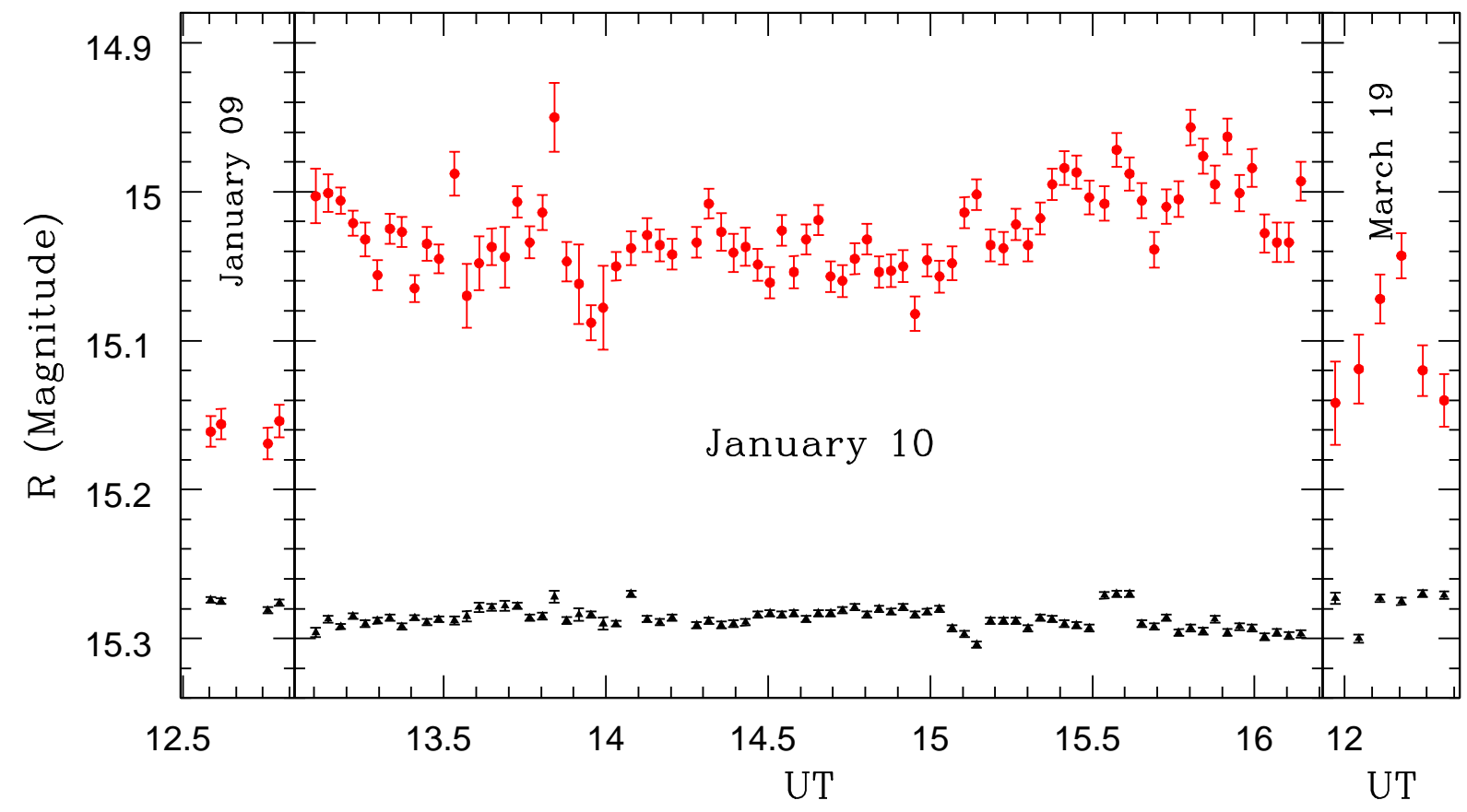

Fig. 1. - R band light curve of AO 0235+164 (filled circles) and differential instrumental magnitude of standard stars (Star1-Star3) (filled triangles) on the nights January 9, January 10 and March 19, 2007 (from left to right panels) respectively. Standard stars differential light curve is offset for clarity by same arbitrary constant on all the three nights of observations. 

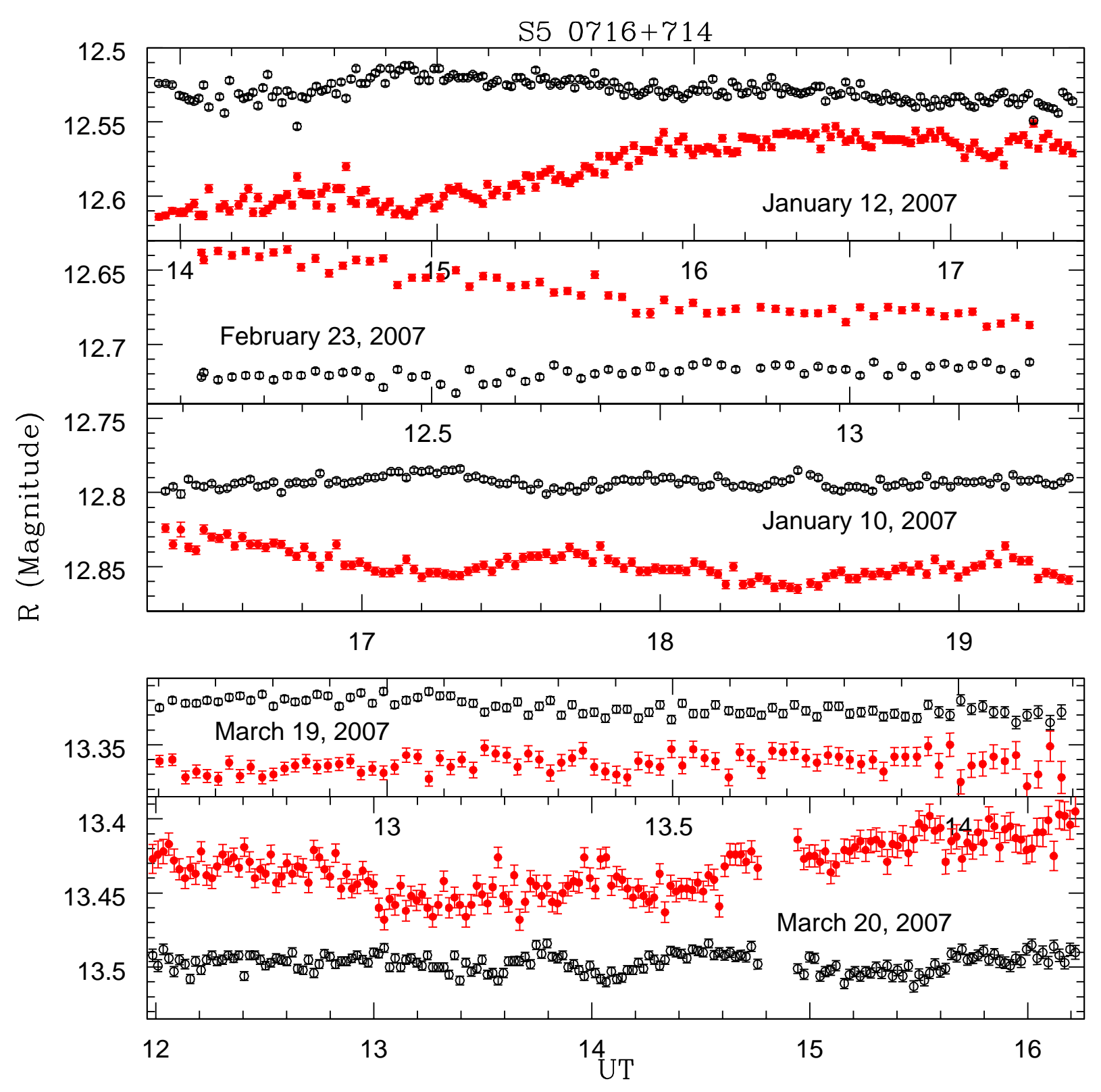

Fig. 2.- R band light curve of S5 0716+714 (filled circles) and differential instrumental magnitude of standard stars (Star5-Star3) (open circles) on the nights January 12, February 23, January 10, March 19 and March 202007 (from top to bottom panels) respectively. Standard stars differential light curve is offset for clarity by different arbitrary constants on the 5 nights of observations. 


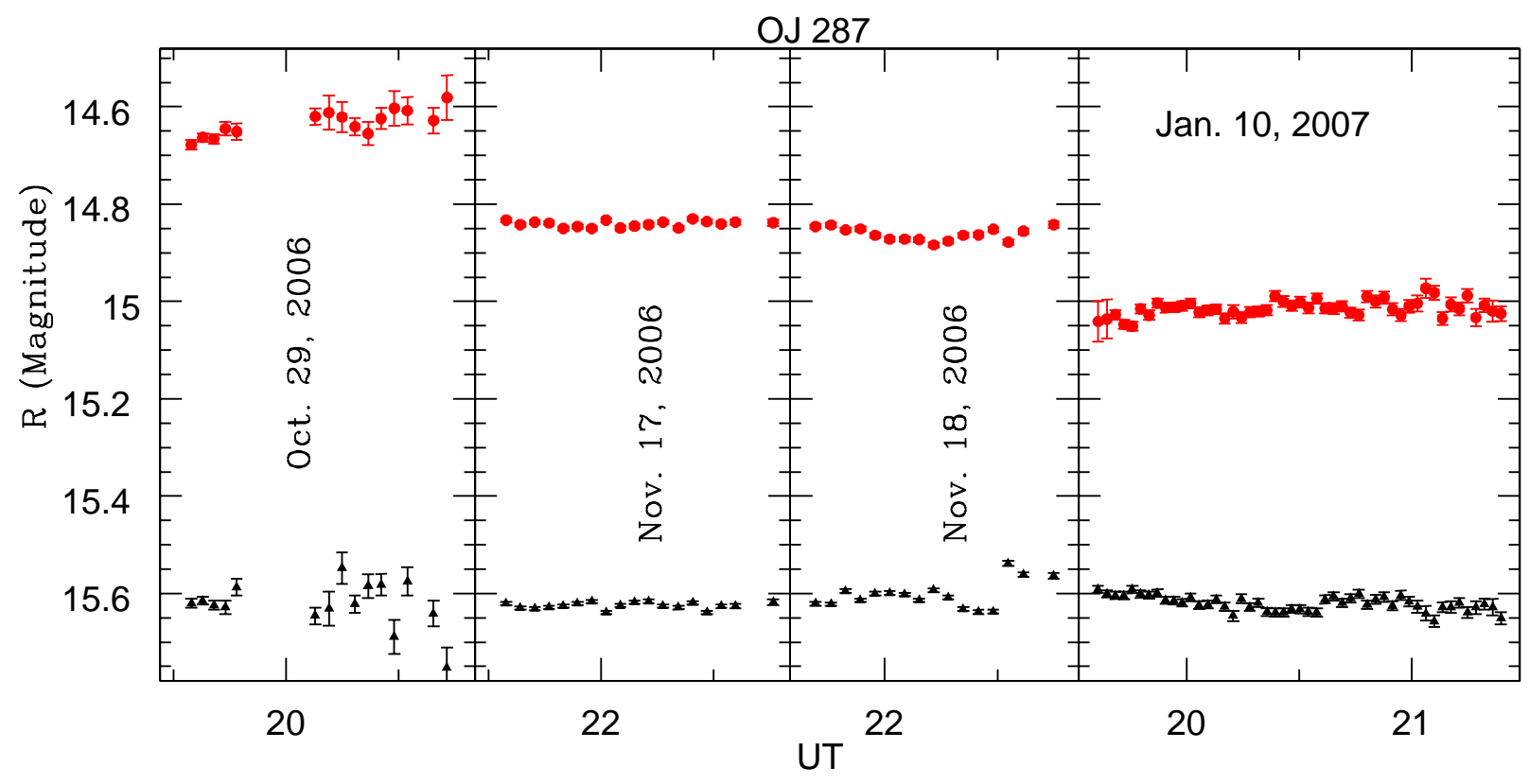

Fig. 3.- R band light curve of OJ 287 (filled circles) and differential instrumental magnitude of standard stars (Star10-Star11) (filled triangles) on the nights October 29, November 17, November 18, 2006 and January 10, 2007 (from left to right panels) respectively. Standard stars differential light curve is offset for clarity by same arbitrary constant on the all 4 nights of observations. 


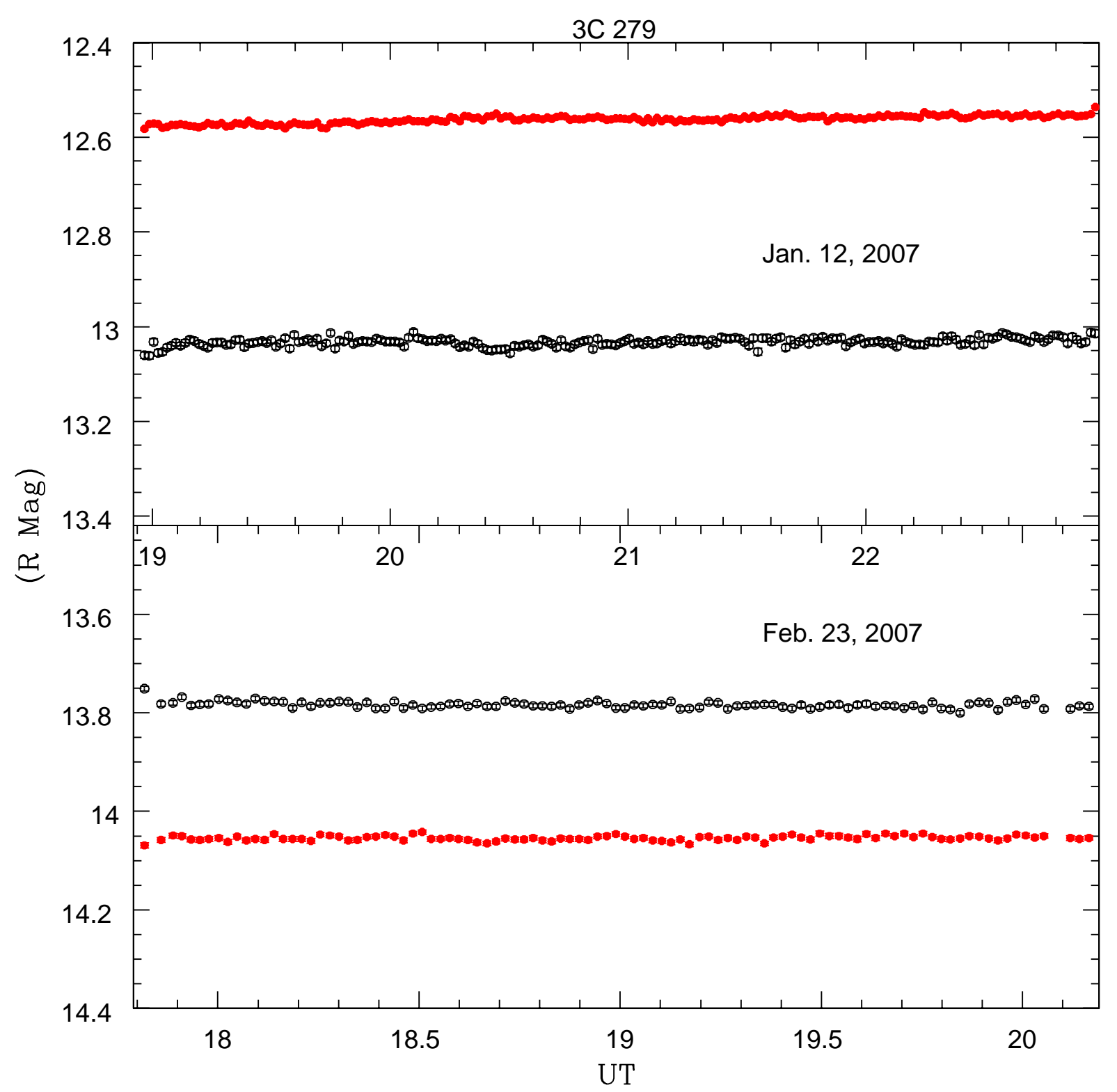

Fig. 4. - R band light curve of 3C 279 (filled circles) and differential instrumental magnitude of standard stars (Star8-Star1) (open circles) on the nights of January 12 (top panel) and February 23, 2007 (bottom panel). Standard stars differential light curve is offset for clarity by different arbitrary constants on both the nights of observations. 


\section{$3 \mathrm{C} 454.3$}
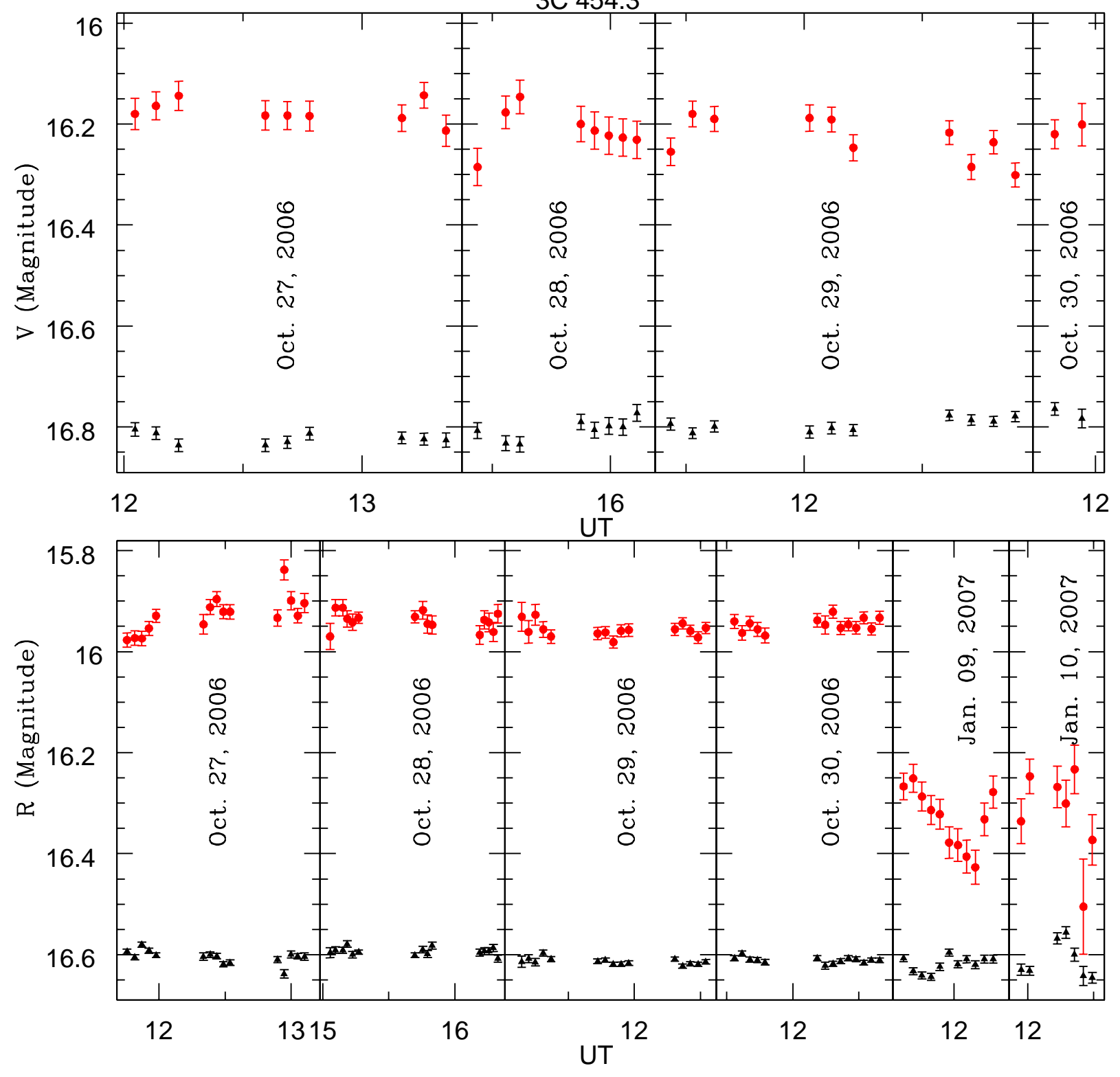

Fig. 5.- V and R bands light curve of 3C 454.3 (filled circles) and differential instrumental magnitude of standard stars (Star3-Star4) (filled triangles) on panels in top row and panels in bottom row respectively. Date of observations are marked in individual panels. Standard stars differential light curve is offset for clarity by the one arbitrary constants for $\mathrm{R}$ and another arbitrary constants for $\mathrm{V}$ on all the nights of observations. 


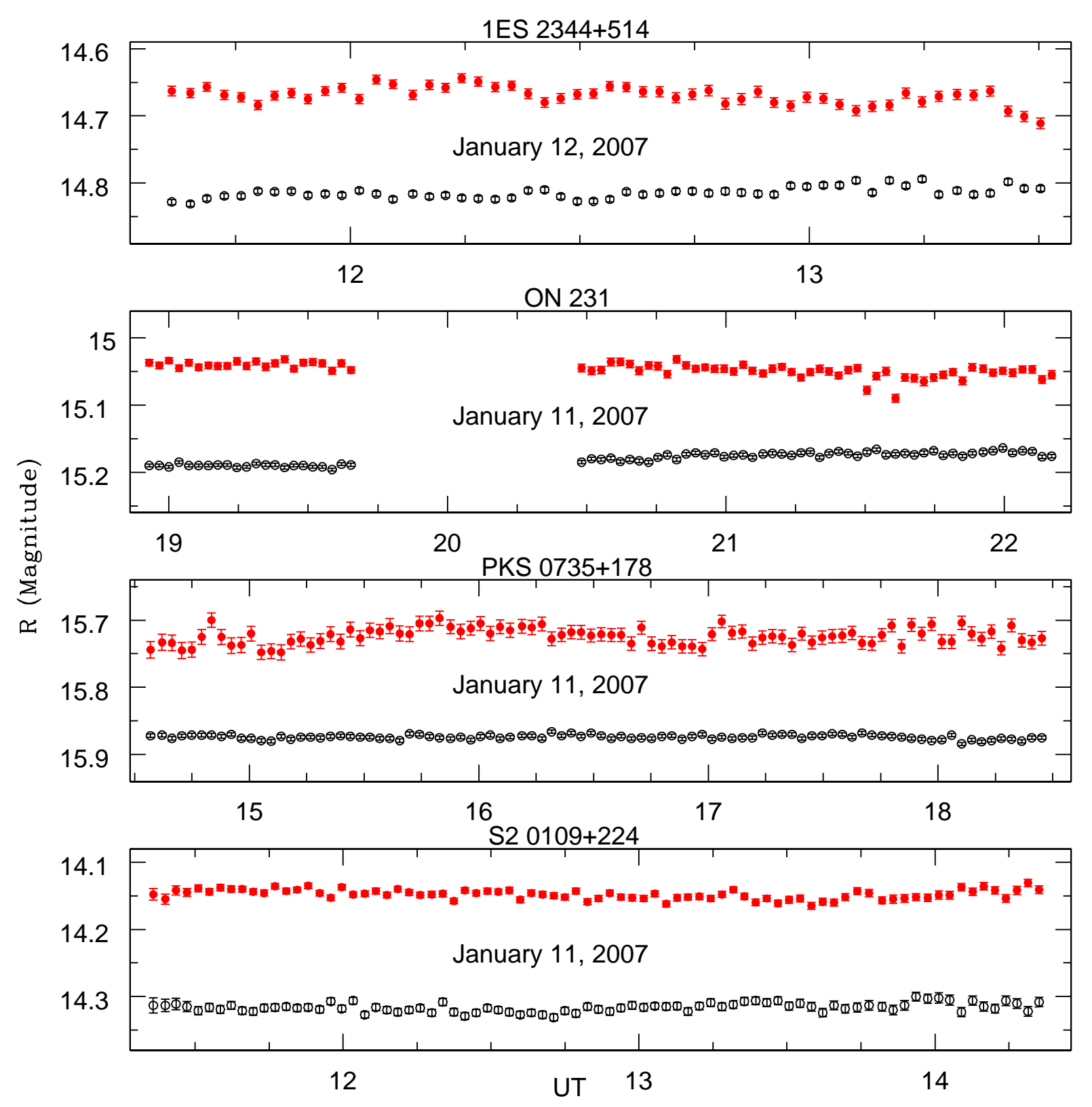

Fig. 6. - R band light curves of S2 0109+224, PKS 0735+178, ON 231 and 1ES 2344+514 (filled circles) from bottom to top panels respectively. For S2 0109+224, differential instrumental magnitude of standard stars (Star D - Star C1); for PKS 0735+178, differential instrumental magnitude of standard stars (Star C4 - Star C1); for ON 231, differential instrumental magnitude of standard stars (Star D - Star A); for 1ES 2344+514, differential instrumental magnitude of standard stars (Star C2 - Star C1) are plotted by (open circles) for clarity by using arbitrary constants. 

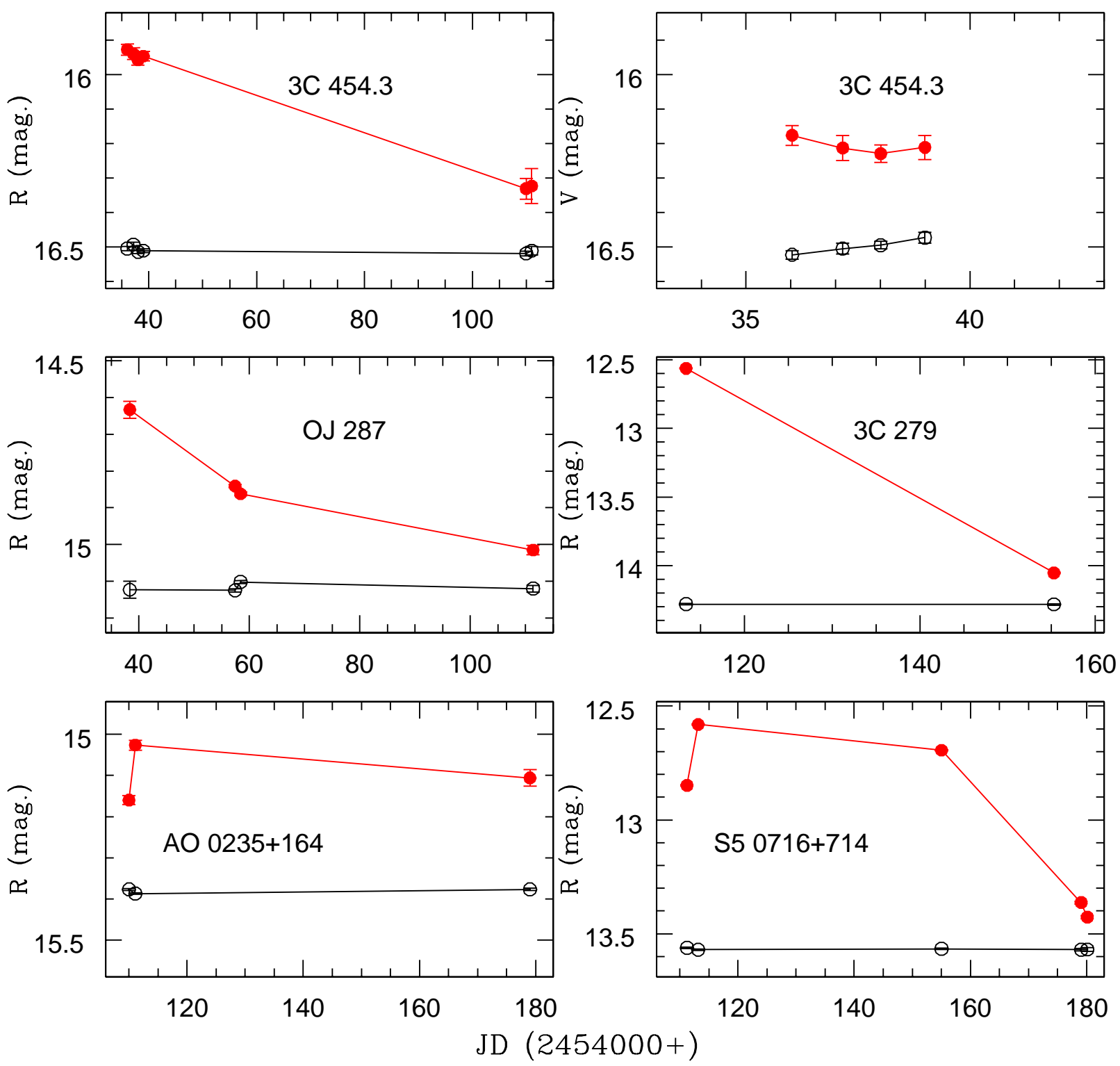

Fig. 7. - The figure show the average $\mathrm{R}$ band magnitudes of the blazars AO $0235+164$, S5 0716+714, OJ 287, 3C 279 and 3C 454.3 (between October 27, 2006 to March 20, 2007). Top left panel of the figure shows the average $\mathrm{V}$ band magnitude of the blazar 3C 454.3 in 4 nights (October 27-30, 2006). Average magnitude of blazars are shown by filled circles. Open circles show the differential instrumental magnitudes of two standard stars in the blazar field offset by same arbitrary constant for specific blazar on all observing nights for clarity. 